\title{
Existence and multiplicity of solutions for fractional Hamiltonian systems
}

\author{
Guoqing Chai ${ }^{1 *}$ and Weiming Liu ${ }^{1}$
}

\author{
"Correspondence: \\ guoqingchai@hbnu.edu.cn \\ ${ }^{1}$ College of Mathematics and \\ Statistics, Hubei Normal University, \\ Huangshi, P.R. China
}

\begin{abstract}
In this paper, the authors investigate the existence and multiplicity of solutions for the following fractional Hamiltonian system:

$$
\left\{\begin{array}{l}
{ }_{t} D_{\infty}^{\alpha}\left({ }_{-\infty} D_{t}^{\alpha} u(t)\right)+V(t) u(t)=\lambda u(t)+b(t)|u(t)|^{q-2} u(t)+\mu h(t), \quad t \in \mathbb{R} \\
u \in H^{\alpha}\left(\mathbb{R}, \mathbb{R}^{N}\right)
\end{array}\right.
$$

where $\alpha \in(1 / 2,1), q>2, b \in C(\mathbb{R},(0, \infty)), h \in C\left(\mathbb{R}, \mathbb{R}^{N}\right), N \geq 1, \lambda, \mu$ are parameters, and $V \in C\left(\mathbb{R}, \mathbb{R}^{N \times N}\right)$ is a positive definite symmetry matrix for all $t \in \mathbb{R}$. By using the variational method and with the help of the Nehari manifold, the existence results of at least one or two nontrivial solutions to the above fractional Hamiltonian system are obtained.
\end{abstract}

Keywords: Fractional Hamiltonian systems; Critical point; Variational method; Nehari manifold

\section{Introduction}

A large number of studies have shown that fractional differential equations have many applications in science and engineering [1-5]. Compared with integer differential equations, fractional differential equations can more effectively and accurately simulate various natural phenomena, such as in neurons, economics, image processing, viscoelasticity, biochemical processes, bioengineering, electromagnetic phenomena, etc. Because of the importance of fractional equations in theory and application, in the past decade, researchers dealing with fractional differential equations have achieved significant development. By applying the fixed point theory, the topology theory, and the monotonically iterative techniques, a lot of results on the existence and multiplicity of solutions have been established [6-9]. In recent years, the study of fractional differential equations by variational methods has attracted researchers' great attention [10-31]. This is an interesting and new research trend. Especially, for the fractional differential equations involving both the left and right fractional derivatives, in general, the fixed point theory is not suitable for studying the existence to this kind of equations because it is not easy to convert this kind of fractional equation into an equivalent integral equation and then transform it into some fixed point problem of an operator. For the first time, Jiao and Zhou in [10] show that the variational method is a very effective way to study such problems. By establishing some appropriate

(c) The Author(s) 2019. This article is distributed under the terms of the Creative Commons Attribution 4.0 International License (http://creativecommons.org/licenses/by/4.0/), which permits unrestricted use, distribution, and reproduction in any medium, provided you give appropriate credit to the original author(s) and the source, provide a link to the Creative Commons license, and indicate if changes were made. 
spaces and variational structure, the authors successfully apply the critical point theory to the following problems of fractional differential equations:

$$
\left\{\begin{array}{l}
{ }_{t} D_{T}^{\alpha}\left({ }_{0} D_{t}^{\alpha} u(t)\right)=\nabla F(t, u(t)), \quad \text { a.e. } t \in[0, T], \\
u(0)=u(T)=0 .
\end{array}\right.
$$

Under some suitable conditions, the existence results have been obtained.

Recently, in [24], motivated by the work in [10] mentioned above, Torres investigates a kind of fractional Hamiltonian systems with the left and right fractional derivatives on $\mathbb{R}$ as follows:

$$
{ }_{t} D_{\infty}^{\alpha}\left(-\infty D_{t}^{\alpha} u(t)\right)+L(t) u(t)=\nabla W(t, u(t))
$$

where $\alpha \in(1 / 2,1), t \in \mathbb{R}, u \in \mathbb{R}^{N}, L \in C\left(\mathbb{R}, \mathbb{R}^{N \times N}\right)$ is a symmetry matrix-valued function satisfying the following condition:

(L1) $L(t)$ is a positive definite symmetric matrix for all $t \in \mathbb{R}$, and there is $l \in C(\mathbb{R},(0, \infty))$ such that $l(t) \rightarrow \infty$ as $t \rightarrow \infty$ as well as

$$
(L(t) x, x) \geq l(t)|x|^{2} \quad \text { for any } t \in \mathbb{R}, x \in \mathbb{R}^{N} .
$$

Under condition (L1) and some other conditions, applying the mountain pass theorem, the author obtained the existence result to problem (1.2).

For the above system (1.2), if $\alpha=1$, then it will reduce to the following second order Hamiltonian system:

$$
u^{\prime \prime}-L(t) u+\nabla W(t, u)=0 .
$$

Poincare [32] was first to recognize the existence of homoclinic solutions of Hamiltonian systems (1.3) and its importance for studying the behaving on dynamical systems. Following this existence, one can infer the existence of chaos nearby or the bifurcation behavior of periodic orbits. In the past two decades, variational methods and critical point theory have been successfully applied to research the existence and multiplicity of homoclinic solutions [33, 34].

Continuing the study in [24], Amado and Torres further investigate problem (1.2). Under some weaker condition than (L1) as well as some conditions, the authors have obtained infinitely many solutions to problems via the genus properties in the critical point theory [25]. For more research on problem (1.2), the readers can refer to [26-29, 35, 36] and the references therein.

Motivated by the works mentioned above, in this paper the authors consider the following fractional Hamiltonian system:

$$
\left\{\begin{array}{l}
{ }_{t} D_{\infty}^{\alpha}\left({ }_{-} D_{t}^{\alpha} u(t)\right)+V(t) u(t)=\lambda u(t)+b(t)|u(t)|^{q-2} u(t)+\mu h(t), \quad t \in \mathbb{R}, \\
u \in H^{\alpha}\left(\mathbb{R}, \mathbb{R}^{N}\right),
\end{array}\right.
$$

where $\alpha \in(1 / 2,1), q>2, N \geq 1, b \in C(\mathbb{R},(0, \infty)), h \in C\left(\mathbb{R}, \mathbb{R}^{N}\right), \lambda, \mu$ are parameters, $V \in$ $C\left(\mathbb{R}, \mathbb{R}^{N \times N}\right)$ is a positive definite symmetry matrix for all $t \in \mathbb{R}$. Indeed, system (1.4) is a 
class of quite important fractional Hamiltonian systems with the perturbed terms $\lambda u$ and $\mu h$. If $\alpha=1,(1.4)$ reduces to the following classical second order Hamiltonian system:

$$
\left\{\begin{array}{l}
u^{\prime \prime}(t)+V(t) u(t)=\lambda u(t)+b(t)|u(t)|^{q-2} u(t)+\mu h(t), \quad t \in \mathbb{R}, \\
u \in H^{1}\left(\mathbb{R}, \mathbb{R}^{N}\right),
\end{array}\right.
$$

which is a prototype of many problems. For more information, readers can refer to [37].

Because the energy functional corresponding to system (1.4) is unbounded from below, it is invalid to try finding a critical point by applying the direct method of minimization to the energy functional. To overcome this difficulty, we introduce the Nehari manifold $N_{\lambda, \mu}$ and split it into three parts $N_{\lambda, \mu}^{-}, N_{\lambda, \mu}^{+}$, and $N_{\lambda, \mu}^{0}$. By looking for minimizer of the energy functional on $N_{\lambda, \mu}^{-}$and $N_{\lambda, \mu}^{+}$respectively and with the help of more analysis techniques, the authors establish some results on the existence and multiplicity of solutions to problem (1.4).

The remaining part of this paper is organized as follows. In Sect. 2, we give some necessary definitions and suitable variational work frame for (1.4). Finally, we will establish the results on the existence of solutions to (1.4) in Sect. 3.

\section{Preliminaries}

In this section, we introduce some basic conceptions of fractional calculus and fractional space.

Definition 2.1 ([3]) The left and right Liouville-Weyl fractional integrals of order $0<\alpha<$ 1 for function $u$ are defined as

$$
-\infty I_{t}^{\alpha} u(t)=\frac{1}{\Gamma(\alpha)} \int_{-\infty}^{t}(t-s)^{\alpha-1} u(s) d s
$$

and

$$
{ }_{t} I_{\infty}^{\alpha} u(t)=\frac{1}{\Gamma(\alpha)} \int_{t}^{\infty}(s-t)^{\alpha-1} u(s) d s
$$

respectively.

Definition 2.2 ([3]) The left and right Liouville-Weyl fractional derivatives of order $0<$ $\alpha<1$ for function $u$ are defined as

$$
{ }_{-\infty} D_{t}^{\alpha} u(t)=\frac{d}{d t}-\infty I_{t}^{1-\alpha} u(t)
$$

and

$$
{ }_{t} D_{\infty}^{\alpha} u(t)=-\frac{d}{d t}{ }_{t} I_{\infty}^{1-\alpha} u(t)
$$

respectively.

On the Liouville-Weyl fractional derivative, we have the following relations:

$$
{ }_{-\infty} D_{t}^{\alpha} u(t)=\frac{\alpha}{\Gamma(1-\alpha)} \int_{0}^{\infty} \frac{u(t)-u(t-s)}{s^{\alpha+1}} d s,
$$




$$
{ }_{t} D_{\infty}^{\alpha} u(t)=\frac{\alpha}{\Gamma(1-\alpha)} \int_{0}^{\infty} \frac{u(t)-u(t+s)}{s^{\alpha+1}} d s .
$$

Moreover, we introduce the Fourier transform $\hat{u}(w)$ of $u(t)$ as follows:

$$
\hat{u}(w)=\int_{-\infty}^{\infty} e^{-i w t} u(t) d t, \quad w \in \mathbb{R},
$$

which satisfies

$$
\begin{array}{ll}
\widehat{-\infty} \widehat{I_{t}^{\alpha} u}(t)(w)=(i w)^{-\alpha} \hat{u}(w), & \widehat{{ }_{t} \widehat{I_{\infty}^{\alpha} u}}(t)(w)=(-i w)^{-\alpha} \hat{u}(w), \\
{ }_{-\infty} \widehat{D_{t}^{\alpha} u}(t)(w)=(i w)^{\alpha} \hat{u}(w), & { }_{t} \widehat{D_{\infty}^{\alpha} u}(t)(w)=(-i w)^{\alpha} \hat{u}(w) .
\end{array}
$$

Now, we introduce some fractional derivative space; for more details, refer to [38].

As usual, for $1 \leq p<\infty$, denote by $L^{P}\left(\mathbb{R}, \mathbb{R}^{N}\right)$ the Banach spaces of functions under the norm $\|u\|_{L^{P}}=\left(\int_{\mathbb{R}}|u(t)|^{P} d t\right)^{\frac{1}{P}} \cdot L^{\infty}\left(\mathbb{R}, \mathbb{R}^{N}\right)$ is the Banach spaces of essentially bounded functions on the norm $\|u\|_{\infty}=\operatorname{ess} \sup \{|u(t)|: t \in \mathbb{R}\}$.

For $\alpha>0$, define the semi-norm $|u|_{I_{-\infty}^{\alpha}}=\left\|_{-\infty} D_{t}^{\alpha} u\right\|_{L^{2}}$ and the norm

$$
\|u\|_{I_{-\infty}^{\alpha}}=\left(|u|_{I_{-\infty}^{\alpha}}^{2}+\|u\|_{L^{2}}^{2}\right)^{\frac{1}{2}}
$$

Let $I_{-\infty}^{\alpha}$ be the closure of space $C_{0}^{\infty}\left(\mathbb{R}, \mathbb{R}^{N}\right)$ on the norm $\|\cdot\|_{I_{-\infty}^{\alpha}}$. Moreover, for $0<\alpha<1$, define the semi-norm $|u|_{\alpha}=\left\||w|^{\alpha} \hat{u}\right\|_{L^{2}}$ and the norm

$$
\|u\|_{\alpha}=\left(|u|_{\alpha}^{2}+\|u\|_{L^{2}}^{2}\right)^{\frac{1}{2}} .
$$

Denote by $H^{\alpha}$ the closure of space $C_{0}^{\infty}\left(\mathbb{R}, \mathbb{R}^{N}\right)$ on the norm $\|\cdot\|_{\alpha}$.

Noting that a function $u \in L^{2}\left(\mathbb{R}, \mathbb{R}^{N}\right)$ belongs to $I_{-\infty}^{\alpha}$ if and only if $|w|^{\alpha} \hat{u} \in L^{2}\left(\mathbb{R}, \mathbb{R}^{N}\right)$. Moreover, $|u|_{I_{-\infty}^{\alpha}}=\left\||w|^{\alpha} \hat{u}\right\|_{L^{2}}$, the spaces $H^{\alpha}$ and $I_{-\infty}^{\alpha}$ are equivalent on the corresponding semi-norms as well as norms.

Denote by $C\left(\mathbb{R}, \mathbb{R}^{N}\right)$ the space of continuous functions with norm $\|u\|_{\infty}:=\sup _{t \in \mathbb{R}}|u(t)|$. We have the following lemma.

Lemma 2.1 ([24]) If $\alpha>1 / 2$, then $H^{\alpha} \subset C\left(\mathbb{R}, \mathbb{R}^{N}\right)$ and there exists a constant $C_{\alpha}$ such that $\|u\|_{\infty} \leq C_{\alpha}\|u\|_{\alpha}$.

It follows from Lemma 2.1 that if $\alpha \in(1 / 2,1)$, then $H^{\alpha} \subset L^{p}\left(\mathbb{R}, \mathbb{R}^{N}\right)$ for any $p \in[2, \infty]$, noting that $\int_{\mathbb{R}}|u(t)|^{p} d t \leq\|u\|_{\infty}^{p-2}\|u\|_{L^{2}}^{2}$.

We need to introduce some variational framework to investigate the existence of solutions to problem (1.4).

First, we give a condition as follows.

$\left(V_{0}\right)$ For each $t \in \mathbb{R}, V(t)$ is a positive definite symmetric matrix. Moreover, there is a function $v_{0} \in C(\mathbb{R},(0, \infty))$ satisfying that $v_{0}(t) \rightarrow \infty$ as $|t| \rightarrow \infty$ as well as

$$
(V(t) u, u) \geq v_{0}(t)|u|^{2}, \quad \text { for any } t \in \mathbb{R}, u \in \mathbb{R}^{N} .
$$


We also need another fractional space. Let

$$
X^{\alpha}=\left\{u \in H^{\alpha}: \int_{\mathbb{R}}\left[\left|{ }_{-\infty} D_{t}^{\alpha} u(t)\right|^{2}+(V(t) u(t), u(t))\right] d t<\infty\right\},
$$

then by [24], $X^{\alpha}$ is a reflexive and separable Hilbert space with the inner product

$$
\langle u, v\rangle_{X^{\alpha}}=\int_{\mathbb{R}}\left[\left({ }_{-\infty} D_{t}^{\alpha} u(t),{ }_{-\infty} D_{t}^{\alpha} v(t)\right)+(V(t) u(t), u(t))\right] d t
$$

and a Banach space with respect to the norm $\|u\|_{X^{\alpha}}=\sqrt{\langle u, v\rangle_{X^{\alpha}}}$.

The following embedding theorem is important to seek some minimum point of a function on the Nehari manifold.

Lemma 2.2 ([24]) Under condition $\left(V_{0}\right), X^{\alpha}$ is continuously embedded in $H^{\alpha}$.

Remark 2.1 By Lemmas 2.1-2.2, there exists a constant $C_{\infty}>0$ such that $\|u\|_{\infty} \leq$ $C_{\infty}\|u\|_{X^{\alpha}}$ for all $u \in X^{\alpha}$.

Lemma 2.3 ([24]) Under condition $\left(V_{0}\right)$, the embedding of $X^{\alpha}$ in $L^{p}\left(\mathbb{R}, \mathbb{R}^{N}\right)$ is continuous for $p \in[2, \infty]$ and is compact for $p \in[2, \infty)$.

By Lemma 2.3, there exists $C_{p}>0$ such that $\|u\|_{L^{p}} \leq C_{p}\|u\|_{X^{\alpha}}, \forall u \in X^{\alpha}$ for $p \in[2, \infty]$.

The following hypothesis will be used in the sequel.

$\left(H_{1}\right) \quad b \in C(\mathbb{R},(0, \infty)) \cap L^{\infty}, h \in C\left(\mathbb{R}, \mathbb{R}^{N}\right) \cap L^{1}, 1 / 2<\alpha<1$, and $q>2$.

In the following, we always assume that $1 / 2<\alpha<1$ and $q>2$.

Now, we are going to establish a variational framework to investigate the existence of solutions for (1.4) with the help of the Nehari manifold. To this end, we define the functional $I_{\lambda, \mu}: X^{\alpha} \rightarrow \mathbb{R}$ by

$$
I_{\lambda, \mu}(u)=\frac{1}{2}\|u\|_{X^{\alpha}}^{2}-\frac{\lambda}{2} \int_{\mathbb{R}}|u(t)|^{2} d t-\frac{1}{q} \int_{\mathbb{R}} b(t)|u(t)|^{q} d t-\mu \int_{\mathbb{R}} h(t) \cdot u(t) d t .
$$

It is easy to verify that $I_{\lambda, u} \in C^{1}\left(X^{\alpha}, \mathbb{R}\right)$ under conditions $\left(V_{0}\right)$ and $\left(H_{1}\right)$; moreover,

$$
\begin{aligned}
\left\langle I_{\lambda, \mu}^{\prime}(u), v\right\rangle= & \int_{\mathbb{R}}\left[\left({ }_{-} D_{t}^{\alpha} u(t),{ }_{-\infty} D_{t}^{\alpha} v(t)\right)+(V(t) u(t), v(t))\right] d t-\lambda \int_{\mathbb{R}} u(t) \cdot v(t) d t \\
& -\int_{\mathbb{R}} b(t)|u(t)|^{q-2} u(t) \cdot v(t) d t-\mu \int_{\mathbb{R}} h(t) \cdot v(t) d t
\end{aligned}
$$

for $u, v \in X^{\alpha}$.

Owing to the fact that $q>2$, the energy functional $I_{\lambda, \mu}$ is not bounded below on $X^{\alpha}$. We turn to considering the functional on the Nehari manifold

$$
\left.N_{\lambda, \mu}:=\left\{u \in X^{\alpha} \backslash\{0\}|| I_{\lambda, \mu}^{\prime}(u), u\right\rangle=0\right\} .
$$

Obviously, $u \in N_{\lambda, \mu}$ if and only if $u \in X^{\alpha}$ satisfies

$$
\|u\|_{X^{\alpha}}^{2}-\lambda \int_{\mathbb{R}}|u|^{2} d t-\int_{\mathbb{R}} b(t)|u|^{q} d t-\mu \int_{\mathbb{R}} h(t) \cdot u(t) d t=0 .
$$


In order to seek a minimum point for $I_{\lambda, \mu}$, the Nehari manifold $N_{\lambda, \mu}$ needs to be split into three parts:

$$
\begin{aligned}
& \left.N_{\lambda, \mu}^{+}:=\left\{u \in N_{\lambda, \mu}|| J_{\lambda, \mu}^{\prime}(u), u\right\rangle>0\right\}, \\
& N_{\lambda, \mu}^{0}:=\left\{u \in N_{\lambda, \mu}\left|J_{\lambda, \mu}^{\prime}(u), u\right\rangle=0\right\}, \\
& \left.N_{\lambda, \mu}^{-}:=\left\{u \in N_{\lambda, \mu}|| J_{\lambda, \mu}^{\prime}(u), u\right\rangle<0\right\},
\end{aligned}
$$

where $J_{\lambda, \mu}(u)=\left\langle I_{\lambda, \mu}^{\prime}(u), u\right\rangle$. Precisely, we have

$$
\left\langle J_{\lambda, \mu}^{\prime}(u), u\right\rangle=2\|u\|_{X^{\alpha}}^{2}-2 \lambda \int_{\mathbb{R}}|u|^{2} d t-q \int_{\mathbb{R}} b(t)|u|^{q} d t-\mu \int_{\mathbb{R}} h(t) \cdot u(t) d t .
$$

In view of Lemma 2.3 , for $p \in[2, \infty]$, we write

$$
S_{p}=\inf \left\{C>0:\|u\|_{L^{p}} \leq C\|u\|_{X^{\alpha}} \text { for all } u \in X^{\alpha}\right\} .
$$

Let $\lambda_{0}=1 / S_{2}^{2}, b_{\infty}=\sup _{t \in \mathbb{R}} b(t)$. For $-\infty<\lambda<\lambda_{0}$, denote $\mu_{\lambda}$ as

$$
\mu_{\lambda}=\left(b_{\infty} S_{q}^{q}\right)^{\frac{1}{2-q}} \frac{q-2}{\|h\|_{L^{1}} S_{\infty}}\left(\frac{1-\lambda S_{2}^{2}}{q-1}\right)^{\frac{q-1}{q-2}}>0 .
$$

We have the following result.

Lemma 2.4 Assume that hypotheses $\left(V_{0}\right)$ and $\left(H_{1}\right)$ hold. If $-\infty<\lambda<\lambda_{0}$, then $I_{\lambda, \mu}$ is coercive and bounded below on $N_{\lambda, \mu}$.

Proof For any $u \in N_{\lambda, \mu}$, it follows from (2.3) that

$$
\int_{\mathbb{R}} b(t)|u|^{q} d t=\|u\|_{X^{\alpha}}^{2}-\lambda \int_{\mathbb{R}}|u|^{2} d t-\mu \int_{\mathbb{R}} h(t) \cdot u(t) d t .
$$

Therefore, by (2.1) and (2.5), we have

$$
\begin{aligned}
I_{\lambda, u}(u) & =\left(\frac{1}{2}-\frac{1}{q}\right)\left(\|u\|_{X^{\alpha}}^{2}-\lambda \int_{\mathbb{R}}|u|^{2} d t\right)-\mu\left(1-\frac{1}{q}\right) \int_{\mathbb{R}} h(t) u(t) d t \\
& \geq\left(\frac{1}{2}-\frac{1}{q}\right)\left(1-\lambda S_{2}^{2}\right)\|u\|_{X^{\alpha}}^{2}-\mu\left(1-\frac{1}{q}\right) \int_{\mathbb{R}}|h(t) \| u(t)| d t \\
& \geq\left(\frac{1}{2}-\frac{1}{q}\right)\left(1-\lambda S_{2}^{2}\right)\|u\|_{X^{\alpha}}^{2}-\mu\left(1-\frac{1}{q}\right)\|h\|_{L^{1}}\|u\|_{\infty} \\
& \geq\left(\frac{1}{2}-\frac{1}{q}\right)\left(1-\lambda S_{2}^{2}\right)\|u\|_{X^{\alpha}}^{2}-\mu\left(1-\frac{1}{q}\right)\|h\|_{L^{1}} S_{\infty}\|u\|_{X^{\alpha}} .
\end{aligned}
$$

Hence, owing to the fact that $\lambda<\lambda_{0}$ and $q>2, I_{\lambda, \mu}$ is coercive and therefore bounded below on $N_{\lambda, \mu}$.

Lemma 2.5 Assume that hypotheses $\left(V_{0}\right)$ and $\left(H_{1}\right)$ hold. If $u_{0} \in N_{\lambda, \mu}$ is a local minimizer of $I_{\lambda, \mu}$ on $N_{\lambda, \mu}$ with $u_{0} \notin N_{\lambda, \mu}^{0}$, then $u_{0}$ is a nontrivial solution to problem (1.4). 
Proof Because $u_{0} \in N_{\lambda, \mu}$ is a local minimizer of $I_{\lambda, \mu}$ on $N_{\lambda, \mu}$, by Theorem 4.1.1 in [39], there exists a number $\eta$ such that

$$
I_{\lambda, \mu}^{\prime}\left(u_{0}\right)=\eta J_{\lambda, \mu}^{\prime}\left(u_{0}\right)
$$

where $J_{\lambda, \mu}(u)=I_{\lambda, \mu}^{\prime}(u) u$. Thus, from $u_{0} \in N_{\lambda, \mu}$ it follows that

$$
0=I_{\lambda, \mu}^{\prime}\left(u_{0}\right) u_{0}=\eta J_{\lambda, \mu}^{\prime}\left(u_{0}\right) u_{0}
$$

In view of $u_{0} \notin N_{\lambda, \mu}^{0}$, we obtain $J_{\lambda, \mu}^{\prime}\left(u_{0}\right) u_{0} \neq 0$, and therefore $\eta=0$. Thus, $I_{\lambda, \mu}^{\prime}\left(u_{0}\right)=0$ follows from (2.7). It means that $u_{0}$ is a nontrivial solution to problem (1.4).

Lemma 2.6 Assume that hypotheses $\left(V_{0}\right)$ and $\left(H_{1}\right)$ hold. If $h=0$, then $N_{\lambda, \mu}^{0}=\emptyset ;$ If $h \neq 0$, then $N_{\lambda, \mu}^{0}=\emptyset$ for $-\infty<\lambda<\lambda_{0}, 0<\mu<\mu_{\lambda}$, where $\mu_{\lambda}$ as in (2.6).

Proof In fact, if not, then there exists $u_{0} \in N_{\lambda, \mu}^{0}$ with $u_{0} \neq 0$. Then, by (2.3)-(2.4), we have

$$
\begin{aligned}
& \left\|u_{0}\right\|_{X^{\alpha}}^{2}-\lambda \int_{\mathbb{R}}\left|u_{0}\right|^{2} d t-\int_{\mathbb{R}} b(t)\left|u_{0}\right|^{q} d t-\mu \int_{\mathbb{R}} h(t) \cdot u_{0}(t) d t=0, \\
& 2\left\|u_{0}\right\|_{X^{\alpha}}^{2}-2 \lambda \int_{\mathbb{R}}\left|u_{0}\right|^{2} d t-q \int_{\mathbb{R}} b(t)\left|u_{0}\right|^{q} d t-\mu \int_{\mathbb{R}} h(t) \cdot u_{0}(t) d t=0 .
\end{aligned}
$$

(i) If $h=0$, then by (2.8)-(2.9), we obtain $\int_{\mathbb{R}} b(t)\left|u_{0}\right|^{q} d t=0$, which is a contradiction noting that $u_{0} \neq 0$ and $b \in C(\mathbb{R},(0, \infty))$.

(ii) If $h \neq 0$, then by subtracting (2.8) from (2.9), we have

$$
\left\|u_{0}\right\|_{X^{\alpha}}^{2}-\lambda \int_{\mathbb{R}}\left|u_{0}\right|^{2} d t-(q-1) \int_{\mathbb{R}} b(t)\left|u_{0}\right|^{q} d t=0 .
$$

Multiplying (2.8) by $q$ and subtracting (2.9), we get

$$
(q-2)\left(\left\|u_{0}\right\|_{X^{\alpha}}^{2}-\lambda \int_{\mathbb{R}}\left|u_{0}\right|^{2} d t\right)-\mu(q-1) \int_{\mathbb{R}} h(t) \cdot u_{0}(t) d t=0 .
$$

Thus, by (2.10)-(2.11) together with (2.5), we have respectively

$$
\left(1-\lambda S_{2}^{2}\right)\left\|u_{0}\right\|_{X^{\alpha}}^{2} \leq(q-1) b_{\infty} \int_{\mathbb{R}}\left|u_{0}\right|^{q} d t \leq(q-1) b_{\infty} S_{q}^{q}\left\|u_{0}\right\|_{X^{\alpha}}^{q}
$$

and

$$
(q-2)\left(1-\lambda S_{2}^{2}\right)\left\|u_{0}\right\|_{X^{\alpha}}^{2} \leq \mu(q-1)\|h\|_{L^{1}}\left\|u_{0}\right\|_{\infty} \leq \mu(q-1)\|h\|_{L^{1}} S_{\infty}\left\|u_{0}\right\|_{X^{\alpha}} .
$$

Because $u_{0} \neq 0$ and $\lambda<\lambda_{0}$, from (2.12)-(2.13) it follows that $\mu \geq \mu_{\lambda}$, which contradicts the hypothesis $0<\mu<\mu_{\lambda}$. Thus, $N_{\lambda, \mu}^{0}=\emptyset$. 


\section{Main result}

In this section, we establish some existence results on solutions to problem (1.4). To this end, we first establish several lemmas.

Lemma 3.1 Assume that hypotheses $\left(V_{0}\right)$ and $\left(H_{1}\right)$ hold. If $h=0$, then $N_{\lambda, \mu}^{-} \neq \emptyset$ for $-\infty<$ $\lambda<\lambda_{0}$. If $h \neq 0$, then $N_{\lambda, \mu}^{+} \neq \emptyset$ and $N_{\lambda, \mu}^{-} \neq \emptyset$ for $-\infty<\lambda<\lambda_{0}, 0<\mu<\mu_{\lambda}$, where $\mu_{\lambda}$ as in (2.6).

Proof (i) If $h=0$, then for any $u_{0} \in X^{\alpha} \backslash\{0\}$,

$$
I_{\lambda, \mu}\left(u_{0}\right)=\frac{1}{2}\left\|u_{0}\right\|_{X^{\alpha}}^{2}-\frac{\lambda}{2} \int_{\mathbb{R}}\left|u_{0}\right|^{2} d t-\frac{1}{q} \int_{\mathbb{R}} b(t)\left|u_{0}\right|^{q} d t .
$$

Let $\phi_{u_{0}}(s)=I_{\lambda, \mu}\left(s u_{0}\right)=\frac{s^{2}}{2}\left\|u_{0}\right\|_{X^{\alpha}}^{2}-\frac{\lambda s^{2}}{2} \int_{\mathbb{R}}\left|u_{0}\right|^{2} d t-\frac{s^{q}}{q} \int_{\mathbb{R}} b(t)\left|u_{0}\right|^{q} d t, s \in[0, \infty)$. Then

$$
\phi_{u_{0}}^{\prime}(s)=s\left(\left\|u_{0}\right\|_{X^{\alpha}}^{2}-\lambda \int_{\mathbb{R}}\left|u_{0}\right|^{2} d t\right)-s^{q-1} \int_{\mathbb{R}} b(t)\left|u_{0}\right|^{q} d t, \quad s \in[0, \infty) .
$$

Write $\psi_{u_{0}}(s)=\phi_{u_{0}}^{\prime}(s), s \in[0, \infty)$. Due to that $u_{0} \neq 0, b(t)>0, t \in \mathbb{R}, q>2, \lambda<\lambda_{0}$ and observing that

$$
\left\|u_{0}\right\|_{X^{\alpha}}^{2}-\lambda \int_{\mathbb{R}}|u|^{2} d t \geq\left(1-\lambda S_{2}^{2}\right)\left\|u_{0}\right\|_{X^{\alpha}}^{2}>0
$$

we have that $\psi_{u_{0}}(0)=0, \psi_{u_{0}}(s)>0$ for small $s>0$ and $\psi_{u_{0}}(s) \rightarrow-\infty$ as $s \rightarrow \infty$. Thus there exists unique $\bar{s}>0$ such that

$$
\begin{aligned}
& \psi_{u_{0}}^{\prime}(\bar{s})=0, \quad \psi_{u_{0}}(\bar{s})=\max _{s \geq 0} \psi_{u_{0}}(s)>0, \quad \psi_{u_{0}}^{\prime}(s)>0 \quad \text { for } s \in(0, \bar{s}) \\
& \psi_{u_{0}}^{\prime}(s)<0 \quad \text { for } s \in(\bar{s}, \infty) .
\end{aligned}
$$

In addition, it follows from (3.1) that

$$
\bar{s}=\left(\frac{\left\|u_{0}\right\|_{X^{\alpha}}^{2}-\lambda \int_{\mathbb{R}}\left|u_{0}\right|^{2} d t}{(q-1) \int_{\mathbb{R}} b(t)\left|u_{0}\right|^{q} d t}\right)^{\frac{1}{q-2}}
$$

On the other hand, since $\psi_{u_{0}}(\bar{s})>0, \psi_{u_{0}}^{\prime}(s)<0$ for $s \in(\bar{s}, \infty)$ and $\psi_{u_{0}}(s) \rightarrow-\infty$, there exists unique $s^{-}>\bar{s}$ such that $\psi_{u}\left(s^{-}\right)=0$. Of course, $\psi_{u}^{\prime}\left(s^{-}\right)<0$. Thus, from

$$
\left\langle J_{\lambda, \mu}^{\prime}\left(s u_{0}\right), s u_{0}\right\rangle=\left(\psi_{u_{0}}(s) s\right)^{\prime} s=\psi_{u_{0}}^{\prime}(s) s^{2}+\psi_{u_{0}}(s) s, \quad s \in[0, \infty)
$$

we immediately have

$$
\left\langle J_{\lambda, \mu}^{\prime}\left(s^{-} u_{0}\right), s^{-} u_{0}\right\rangle=\psi_{u_{0}}^{\prime}\left(s^{-}\right)\left(s^{-}\right)^{2}+\psi_{u_{0}}\left(s^{-}\right) s^{-}=\psi_{u_{0}}^{\prime}\left(s^{-}\right)\left(s^{-}\right)^{2}<0
$$

Hence,

$$
s^{-} u_{0} \in N_{\lambda, \mu}^{-}, \quad \phi_{u_{0}}^{\prime}\left(s^{-}\right)=0, \quad \phi_{u_{0}}^{\prime \prime}\left(s^{-}\right)<0 \quad \text { and } \quad \phi_{u_{0}}\left(s^{-}\right)=\sup _{s \geq 0} I_{\lambda, \mu}\left(s u_{0}\right)
$$


noting that $\phi_{u_{0}}^{\prime \prime}=\psi_{u_{0}}^{\prime}$. Thus, $N_{\lambda, \mu}^{-} \neq \emptyset$.

(ii) If $h \neq 0$, then, without loss of generality, we can say that $h(t)=\left(h_{1}, h_{2}, \ldots, h_{N}\right)$ satisfies $h_{1}(t)>0$ for all $t \in\left(t_{0}-r, t_{0}+r\right)$ for some $t_{0} \in \mathbb{R}$ and some $r>0$. Take $\bar{u}=\left(u_{1}, u_{2}, \ldots, u_{N}\right)$ with $u_{k}=0$ for $2 \leq k \leq N$, and $u_{1}(t)$ is given by

$$
u_{1}(t)= \begin{cases}e^{\frac{\left(t-t_{0}\right)^{2}}{\left(t-t_{0}\right)^{2}-r^{2}}} & \text { for }\left|t-t_{0}\right|<r \\ 0 & \text { for }\left|t-t_{0}\right| \geq r\end{cases}
$$

Then $\bar{u} \in C_{0}^{\infty}\left(\mathbb{R}, \mathbb{R}^{N}\right)$, and therefore, $\bar{u} \in X^{\alpha}$ with $\bar{u} \neq 0$. Furthermore, $\int_{\mathbb{R}} h(t) \cdot \bar{u}(t) d t>0$.

In the following, we make an argument similar to that in (i) above.

Let

$$
\begin{aligned}
\phi_{\bar{u}}(s)= & I_{\lambda, \mu}(s \bar{u}) \\
= & \frac{s^{2}}{2}\|\bar{u}\|_{X^{\alpha}}^{2}-\frac{\lambda s^{2}}{2} \int_{\mathbb{R}}|\bar{u}|^{2} d t-\frac{s^{q}}{q} \int_{\mathbb{R}} b(t)|\bar{u}|^{q} d t \\
& -s \mu \int_{\mathbb{R}} h(t) \cdot \bar{u}(t) d t=0, \quad s \in[0, \infty) .
\end{aligned}
$$

Then

$$
\begin{aligned}
& \phi_{\bar{u}}^{\prime}(s)=s\left(\|\bar{u}\|_{X^{\alpha}}^{2}-\lambda \int_{\mathbb{R}}|\bar{u}|^{2} d t\right)-s^{q-1} \int_{\mathbb{R}} b(t)|\bar{u}|^{q} d t-\mu \int_{\mathbb{R}} h(t) \cdot \bar{u}(t) d t, \\
& s \in[0, \infty) .
\end{aligned}
$$

Let $\psi_{\bar{u}}(s)=s\left(\|\bar{u}\|_{X^{\alpha}}^{2}-\lambda \int_{\mathbb{R}}|\bar{u}|^{2} d t\right)-s^{q-1} \int_{\mathbb{R}} b(t)|\bar{u}|^{q} d t$. Then

$$
\psi_{\bar{u}}^{\prime}(s)=\|\bar{u}\|_{X^{\alpha}}^{2}-\lambda \int_{\mathbb{R}}|\bar{u}|^{2} d t-(q-1) s^{q-2} \int_{\mathbb{R}} b(t)|\bar{u}|^{q} d t, \quad s \in[0, \infty) .
$$

Obviously, $\psi_{\bar{u}}(0)=0, \psi_{\bar{u}}(s)>0$ as $s>0$ small enough, and $\psi_{\bar{u}}(s) \rightarrow-\infty$ as $s \rightarrow \infty$. Hence, there exists unique $\bar{s}>0$ such that

$$
\begin{array}{ll}
\psi_{\bar{u}}(\bar{s})=\max _{s \geq 0} \psi_{\bar{u}}(s)>0, \quad \psi_{\bar{u}}^{\prime}(\bar{s})=0, \\
\psi_{\bar{u}}^{\prime}(s)>0 \quad \text { for } s \in(0, \bar{s}) ; \quad \psi_{\bar{u}}^{\prime}(s)<0 \quad \text { for } s \in(\bar{s}, \infty) .
\end{array}
$$

Moreover, by calculation, we get

$$
\begin{aligned}
\psi_{\bar{u}}(\bar{s}) & =\frac{q-2}{q-1}\left(\frac{\left(\|\bar{u}\|_{X^{\alpha}}^{2}-\lambda \int_{\mathbb{R}}|\bar{u}|^{2} d t\right)^{q-1}}{(q-1) \int_{\mathbb{R}} b(t)|\bar{u}|^{q} d t}\right)^{\frac{1}{q-2}} \\
& \geq \frac{q-2}{q-1}\left(\frac{\left(\left(1-\lambda S_{2}^{2}\right)\|\bar{u}\|_{X^{\alpha}}^{2}\right)^{q-1}}{(q-1) b_{\infty} S_{q}^{q}\|\bar{u}\|_{X^{\alpha}}^{q}}\right)^{\frac{1}{q-2}} \\
& =\frac{q-2}{q-1}\left(\frac{\left(1-\lambda S_{2}^{2}\right)^{q-1}}{(q-1) b_{\infty} S_{q}^{q}}\right)^{\frac{1}{q-2}}\|\bar{u}\|_{X^{\alpha}} .
\end{aligned}
$$


Because $0<\mu \int_{\mathbb{R}} h(t) \cdot \bar{u}(t) d t \leq \mu\|h\|_{L^{1}}\|\bar{u}\|_{\infty} \leq \mu\|h\|_{L^{1}} S_{\infty}\|\bar{u}\|_{X^{\alpha}}$, it is easy to verify that $0<\mu \int_{\mathbb{R}} h(t) \cdot \bar{u}(t) d t<\psi_{\bar{u}}(\bar{s})$ as long as $0<\mu<\mu_{\lambda}$, where $\mu_{\lambda}$ is given by (2.6), and therefore, $\phi_{\bar{u}}^{\prime}(\bar{s})>0$ by $(3.4)$.

Now, in terms of (3.4) together with the fact that $\phi_{\bar{u}}^{\prime}(0)<0, \phi_{\bar{u}}^{\prime}(\bar{s})>0$, it follows from (3.6) - (3.7) that there exist unique $0<s^{+}<\bar{s}$ and unique $s_{2}^{-}>\bar{s}$ such that

$$
\begin{aligned}
& \phi_{\bar{u}}^{\prime}\left(s_{1}^{+}\right)=\phi_{\bar{u}}^{\prime}\left(s_{2}^{-}\right)=0, \quad \phi_{\bar{u}}^{\prime \prime}\left(s_{1}^{+}\right)>0, \quad \phi_{\bar{u}}^{\prime \prime}\left(s_{2}^{-}\right)<0 ; \quad \phi_{\bar{u}}^{\prime \prime}(s)>0, \quad s \in(0, \bar{s}) ; \\
& \phi_{\bar{u}}^{\prime \prime}(s)<0, \quad s \in(\bar{s}, \infty)
\end{aligned}
$$

observing that (3.7) together with the fact that $\phi_{\bar{u}}^{\prime}(s)=\psi_{\bar{u}}(s), \phi_{\bar{u}}^{\prime \prime}(s)=\psi_{\bar{u}}^{\prime}(s), s \in[0, \infty)$.

Hence, from

$$
\left\langle J_{\lambda, \mu}^{\prime}(s \bar{u}), s \bar{u}\right\rangle=\left(\phi_{\bar{u}}^{\prime}(s) s\right)^{\prime} s=\phi_{\bar{u}}^{\prime \prime}(s) s^{2}+\phi_{\bar{u}}^{\prime}(s) s,
$$

together with (3.8), we have respectively

$$
\left\langle J_{\lambda, \mu}^{\prime}\left(s_{1}^{+} \bar{u}\right), s_{1}^{+} \bar{u}\right\rangle=\phi_{\bar{u}}^{\prime \prime}\left(s_{1}^{+}\right)\left(s_{1}^{+}\right)^{2}+\phi_{\bar{u}}^{\prime}\left(s_{1}^{+}\right) s_{1}^{+}=\phi_{\bar{u}}^{\prime \prime}\left(s_{1}^{+}\right)\left(s_{1}^{+}\right)^{2}>0
$$

and

$$
\left\langle J_{\lambda, \mu}^{\prime}\left(s_{2}^{-} \bar{u}\right), s_{2}^{-} \bar{u}\right\rangle=\phi_{\bar{u}}^{\prime \prime}\left(s_{2}^{-}\right)\left(s_{2}^{-}\right)^{2}+\phi_{\bar{u}}^{\prime}\left(s_{2}^{-}\right) s_{2}^{-}=\phi_{\bar{u}}^{\prime \prime}\left(s_{2}^{-}\right)\left(s_{2}^{-}\right)^{2}<0,
$$

which means that

$$
s_{1}^{+} \bar{u} \in N_{\lambda, \mu}^{+}, s_{2}^{-} \bar{u} \in N_{\lambda, \mu}^{-}, \quad \phi_{\bar{u}}\left(s_{1}^{+}\right)=\min _{0 \leq s \leq \bar{s}} \phi_{\bar{u}}(s), \quad \phi_{\bar{u}}\left(s_{2}^{-}\right)=\max _{s \geq 0} \phi_{u}(s)
$$

noting that (3.8).

Lemma 3.2 Assume that hypotheses $\left(V_{0}\right)$ and $\left(H_{1}\right)$ hold. If $h \neq 0$, then $-\infty<C_{\lambda, \mu}^{+}:=$ $\inf _{u \in N_{\lambda, \mu}^{+}} I_{\lambda, \mu}<0$ for $-\infty<\lambda<\lambda_{0}, 0<\mu<\mu_{\lambda}$, where $\mu_{\lambda}$ as in (2.6).

Proof First, by Lemma 3.1, $N_{\lambda, \mu}^{+} \neq \emptyset$. For any $u \in N_{\lambda, \mu}^{+}$, we have

$$
0=I_{\lambda, \mu}^{\prime}(u) u=\|u\|_{X^{\alpha}}^{2}-\lambda \int_{\mathbb{R}}|u|^{2} d t-\int_{\mathbb{R}} b(t)|u|^{q} d t-\mu \int_{\mathbb{R}} h(t) \cdot u(t) d t
$$

and

$$
\begin{aligned}
0 & <\left(I_{\lambda, \mu}^{\prime}(u) u\right)^{\prime} u \\
& =2\|u\|_{X^{\alpha}}^{2}-2 \lambda \int_{\mathbb{R}}|u|^{2} d t-q \int_{\mathbb{R}} b(t)|u|^{q} d t-\mu \int_{\mathbb{R}} h(t) \cdot u(t) d t .
\end{aligned}
$$

By (3.10)-(3.11), we have

$$
(q-1) \int_{\mathbb{R}} b(t)|u|^{q} d t<\|u\|_{X^{\alpha}}^{2}-\lambda \int_{\mathbb{R}}|u|^{2} d t
$$


On the other hand, by (2.1) together with (3.10) and (3.12), we have

$$
\begin{aligned}
I_{\lambda, \mu}(u) & =-\frac{1}{2}\left(\|u\|_{X^{\alpha}}^{2}-\lambda \int_{\mathbb{R}}|u|^{2} d t\right)+\frac{q-1}{q} \int_{\mathbb{R}} b(t)|u|^{q} d t \\
& <-\frac{1}{2}\left(\|u\|_{X^{\alpha}}^{2}-\lambda \int_{\mathbb{R}}|u|^{2} d t\right)+\frac{1}{q}\left(\|u\|_{X^{\alpha}}^{2}-\lambda \int_{\mathbb{R}}|u|^{2} d t\right) \\
& =\left(\frac{1}{q}-\frac{1}{2}\right)\left(\|u\|_{X^{\alpha}}^{2}-\lambda \int_{\mathbb{R}}|u|^{2} d t\right)<0
\end{aligned}
$$

noting that $q>2$ and $u \neq 0$. Hence, $C_{\lambda, \mu}^{+}=\inf _{u \in N_{\lambda, \mu}^{+}} I_{\lambda, \mu}(u)<0$.

On the other hand, in terms of Lemma 2.4 and the fact that $I_{\lambda, \mu}$ is bounded below, we obtain that $C_{\lambda, \mu}^{+}>-\infty$.

Lemma 3.3 Assume that hypotheses $\left(V_{0}\right)$ and $\left(H_{1}\right)$ hold. In addition, if

(i) $h=0$, then $C_{\lambda, \mu}^{-}:=\inf _{u \in N_{\lambda, \mu}^{-}} I_{\lambda, \mu}(u)>0$ for $-\infty<\lambda<\lambda_{0}$;

(ii) $h \neq 0$, then $C_{\lambda, \mu}^{-}:=\inf _{u \in N_{\lambda, \mu}^{-}} I_{\lambda, \mu}(u)>0$ for $-\infty<\lambda<\lambda_{0}, 0<\mu<\mu_{\lambda} / 2$, where $\mu_{\lambda}$ as in (2.6).

Proof First, by Lemma 3.1, whether case (i) or case (ii), we always have $N_{\lambda, \mu}^{-} \neq \emptyset$. For any $u \in N_{\lambda, \mu}^{-}$, by $I_{\lambda, \mu}^{\prime}(u) u=0$, we have

$$
\mu \int_{\mathbb{R}} h(t) \cdot u(t) d t=\|u\|_{X^{\alpha}}^{2}-\lambda \int_{\mathbb{R}}|u|^{2} d t-\int_{\mathbb{R}} b(t)|u|^{q} d t
$$

Thus, by $\left(\left(I_{\lambda, \mu}^{\prime} u\right) u\right)^{\prime} u<0$, it follows from (3.13) that

$$
\begin{aligned}
0 & >2\|u\|_{X^{\alpha}}^{2}-2 \lambda \int_{\mathbb{R}}|u|^{2} d t-q \int_{\mathbb{R}} b(t)|u|^{q} d t-\mu \int_{\mathbb{R}} h(t) \cdot u(t) d t \\
& =\|u\|_{X^{\alpha}}^{2}-\lambda \int_{\mathbb{R}}|u|^{2} d t-(q-1) \int_{\mathbb{R}} b(t)|u|^{q} d t,
\end{aligned}
$$

and therefore,

$$
\left(1-\lambda S_{2}^{2}\right)\|u\|_{X^{\alpha}}^{2} \leq\|u\|_{X^{\alpha}}^{2}-\lambda \int_{\mathbb{R}}|u|^{2} d t<(q-1) \int_{\mathbb{R}} b(t)|u|^{q} d t \leq(q-1) b_{\infty} S_{q}^{q}\|u\|_{X^{\alpha}}^{q}
$$

Thus,

$$
\|u\|_{X^{\alpha}}>\left(\frac{\left(1-\lambda S_{2}^{2}\right)}{(q-1) b_{\infty} S_{q}^{q}}\right)^{\frac{1}{q-2}}:=d_{0}>0
$$

On the other hand, by (2.1) together with (3.13) as well as (2.5), we have

$$
\begin{aligned}
I_{\lambda, \mu}(u) & =\left(\frac{1}{2}-\frac{1}{q}\right)\left(\|u\|_{X^{\alpha}}^{2}-\lambda \int_{\mathbb{R}}|u|^{2} d t\right)-\mu\left(1-\frac{1}{q}\right) \int_{\mathbb{R}} h(t) \cdot u(t) d t \\
& \geq\left(\frac{1}{2}-\frac{1}{q}\right)\left(1-\lambda S_{2}^{2}\right)\|u\|_{X^{\alpha}}^{2}-\mu\left(1-\frac{1}{q}\right) S_{\infty}\|h\|_{L^{1}}\|u\|_{X^{\alpha}} \\
& =\left[\left(\frac{1}{2}-\frac{1}{q}\right)\left(1-\lambda S_{2}^{2}\right)\|u\|_{X^{\alpha}}-\mu\left(1-\frac{1}{q}\right) S_{\infty}\|h\|_{L^{1}}\right]\|u\|_{X^{\alpha}} .
\end{aligned}
$$


(i) If $h=0$, then by (3.14)-(3.15), we obtain

$$
I_{\lambda, \mu}(u) \geq\left(\frac{1}{2}-\frac{1}{q}\right)\left(1-\lambda S_{2}^{2}\right) d_{0}^{2}>0
$$

and therefore,

$$
\inf _{u \in N_{\lambda, \mu}^{-}} I_{\lambda, \mu}(u) \geq\left(\frac{1}{2}-\frac{1}{q}\right)\left(1-\lambda S_{2}^{2}\right) d_{0}^{2}>0 .
$$

(ii) If $h \neq 0$, then $\|h\|_{L^{1}}>0$. For $0<\mu<\mu_{\lambda} / 2$, by calculation, we can obtain

$$
\begin{aligned}
& \left(\frac{1}{2}-\frac{1}{q}\right)\left(1-\lambda S_{2}^{2}\right)\|u\|_{X^{\alpha}}-\mu\left(1-\frac{1}{q}\right) S_{\infty}\|h\|_{L^{1}} \\
& \geq\left(\frac{1}{2}-\frac{1}{q}\right)\left(1-\lambda S_{2}^{2}\right) d_{0}-\mu\left(1-\frac{1}{q}\right) S_{\infty}\|h\|_{L^{1}}>0
\end{aligned}
$$

and therefore,

$$
I_{\lambda, \mu}(u) \geq\left[\left(\frac{1}{2}-\frac{1}{q}\right)\left(1-\lambda S_{2}^{2}\right) d_{0}-\mu\left(1-\frac{1}{q}\right) S_{\infty}\|h\|_{L^{1}}\right] d_{0}:=d_{1}>0 .
$$

Consequently, $\inf _{u \in N_{\lambda, \mu}^{-}} I_{\lambda, \mu}(u) \geq d_{1}>0$.

Lemma 3.4 Assume that $\left(V_{0}\right),\left(H_{1}\right)$ hold. If $h \neq 0$, then for $-\infty<\lambda<\lambda_{0}, 0<\mu<\mu_{\lambda}$ (where $\mu_{\lambda}$ as in (2.6)), $I_{\lambda, \mu}$ achieves its minimum at some $\bar{u} \in N_{\lambda, \mu}^{+}$on $N_{\lambda, \mu}^{+}$. That is,

$$
I_{\lambda, \mu}(\bar{u})=\inf _{u \in N_{\lambda, \mu}^{+}} I_{\lambda, \mu}(u):=C_{\lambda, \mu}^{+}
$$

Proof First, under conditions $\left(V_{0}\right),\left(H_{1}\right)$ and $h \neq 0$, for $\lambda<\lambda_{0}, 0<\mu<\mu_{\lambda}$, by Lemmas 3.13.2, we know that $N_{\lambda, \mu}^{+} \neq \emptyset$, and $-\infty<C_{\lambda, \mu}^{+}<0$. Let $\left\{u_{n}\right\} \subset N_{\lambda, \mu}^{+}$be a minimizing sequence for $I_{\lambda, \mu}$ on $N_{\lambda, \mu}^{+}$. That is, $\lim _{n \rightarrow \infty} I_{\lambda, \mu}\left(u_{n}\right)=C_{\lambda, \mu}^{+}$. Again, it follows from Lemma 2.4 that $\left\{u_{n}\right\}$ is bounded in $X^{\alpha}$. Thus, by the reflexivity of $X^{\alpha}$, there exists a subsequence, still denoted by $\left\{u_{n}\right\}$, such that $u_{n} \rightarrow \bar{u}$ in $X^{\alpha}$. Thus, by Lemma 2.3 , we have

$$
u_{n} \rightarrow \bar{u} \quad \text { in } L^{q}(\mathbb{R}), p \in[2, \infty), \quad u_{n}(t) \rightarrow \bar{u}(t), \quad \text { a.e. } t \in \mathbb{R} \text {. }
$$

Again, because $\left\{u_{n}\right\}$ is bounded in $X^{\alpha}$, there is a constant $M>0$ such that $\left\|u_{n}\right\|_{X^{\alpha}} \leq M$, $n \geq 1$, and therefore, it follows from (2.5) that

$$
\left|h(t) \cdot u_{n}(t)\right| \leq|h(t)|\left\|u_{n}\right\|_{\infty} \leq|h(t)| S_{\infty}\left\|u_{n}\right\|_{X^{\alpha}} \leq|h(t)| S_{\infty} M \in L^{1}(\mathbb{R}) .
$$

Thus, by applying the dominated convergence theorem, we have

$$
\lim _{n \rightarrow \infty} \int_{\mathbb{R}} h(t) \cdot u_{n}(t) d t=\int_{\mathbb{R}} h(t) \cdot \bar{u}(t) d t
$$

Similarly, we also have

$$
\lim _{n \rightarrow \infty} \int_{\mathbb{R}} b(t)\left|u_{n}(t)\right|^{q} d t=\int_{\mathbb{R}} b(t)|\bar{u}(t)|^{q} d t
$$


Now, we show that $\lim _{n \rightarrow \infty}\left\|u_{n}\right\|_{X^{\alpha}}=\|\bar{u}\|_{X^{\alpha}}$.

In fact, if not, then by the weak lower semi-continuity on norm, we have

$$
\|\bar{u}\|_{X^{\alpha}}<\lim _{n \rightarrow \infty}\left\|u_{n}\right\|_{X^{\alpha}}
$$

We immediately have

$$
I_{\lambda, \mu}(\bar{u})<\lim _{n \rightarrow \infty} I_{\lambda, \mu}\left(u_{n}\right)
$$

by (3.17)-(3.19).

On the other hand, by $u_{n} \in N_{\lambda, \mu}^{+}$, we have

$$
\begin{aligned}
0 & =I_{\lambda, \mu}^{\prime}\left(u_{n}\right) u_{n}=\left\|u_{n}\right\|_{X^{\alpha}}^{2}-\lambda \int_{\mathbb{R}}\left|u_{n}\right|^{2} d t-\int_{\mathbb{R}} b(t)\left|u_{n}\right|^{q} d t-\mu \int_{\mathbb{R}} h(t) \cdot u_{n}(t) d t, \\
0 & <\left(I_{\lambda, \mu}^{\prime}\left(u_{n}\right) u_{n}\right)^{\prime} u_{n} \\
& =2\left\|u_{n}\right\|_{X^{\alpha}}^{2}-2 \lambda \int_{\mathbb{R}}\left|u_{n}\right|^{2} d t-q \int_{\mathbb{R}} b(t)\left|u_{n}\right|^{q} d t-\mu \int_{\mathbb{R}} h(t) \cdot u_{n}(t) d t .
\end{aligned}
$$

By (3.21)-(3.22), we have

$$
\begin{aligned}
(q-1) \mu \int_{\mathbb{R}} h(t) \cdot u_{n}(t) d t & >(q-2)\left(\left\|u_{n}\right\|_{X^{\alpha}}^{2}-\lambda \int_{\mathbb{R}}\left|u_{n}\right|^{2} d t\right) \\
& \geq(q-2)\left(1-\lambda S_{2}^{2}\right)\left\|u_{n}\right\|_{X^{\alpha}}^{2}>0 .
\end{aligned}
$$

Passing to the limit as $n \rightarrow \infty$ on the above inequality, by (3.17) and (3.19), we get

$$
(q-1) \mu \int_{\mathbb{R}} h(t) \cdot \bar{u}(t) d t \geq(q-2)\left(1-\lambda S_{2}^{2}\right) \lim _{n \rightarrow \infty}\left\|u_{n}\right\|_{X^{\alpha}}^{2}>(q-2)\left(1-\lambda S_{2}^{2}\right)\|\bar{u}\|_{X^{\alpha}}^{2} .
$$

Hence, $\int_{\mathbb{R}} h(t) \cdot \bar{u}(t) d t>0$, and therefore, by proof (ii) in Lemma 3.1 and with a derivation similar to (3.8) and (3.9), for $\phi_{\bar{u}}(s)=I_{\lambda, \mu}(s \bar{u}), s \in[0, \infty)$, we know that there exist unique $0<s_{1}^{+}<\bar{s}<s_{2}^{-}$such that

$$
\begin{aligned}
& \phi_{\bar{u}}^{\prime}\left(s_{1}^{+}\right)=\phi_{\bar{u}}^{\prime}\left(s_{2}^{-}\right)=0, \quad s_{1}^{+} \bar{u} \in N_{\lambda, \mu}^{+}, s_{2}^{-} \bar{u} \in N_{\lambda, \mu}^{-}, \\
& \phi_{\bar{u}}\left(s_{1}^{+}\right)=\inf _{0 \leq s \leq \bar{s}} \phi_{\bar{u}}(s), \quad \phi_{\bar{u}}\left(s_{2}^{-}\right)=\sup _{s \geq 0} \phi_{\bar{u}}(s) .
\end{aligned}
$$

Similarly, let $\phi_{u_{n}}(s)=I_{\lambda, \mu}\left(s u_{n}\right)$. Then, by the same reason as (3.8) and (3.9) together with (3.23), from $\int_{\mathbb{R}} h(t) \cdot u_{n}(t) d t>0$, we know that there exist unique $s_{n}^{+}<\bar{s}_{n}<s_{n}^{-}$such that

$$
\phi_{u_{n}}^{\prime}\left(s_{n}^{+}\right)=\phi_{u_{n}}^{\prime}\left(s_{n}^{-}\right)=0, \quad \phi_{u_{n}}^{\prime \prime}(s)>0, \quad s \in\left(0, \bar{s}_{n}\right) ; \quad \phi_{u_{n}}^{\prime \prime}(s)<0, \quad s \in\left(\bar{s}_{n}, \infty\right)
$$

as well as

$$
s_{n}^{+} u_{n} \in N_{\lambda, \mu}^{+}, \quad s_{n}^{-} u_{n} \in N_{\lambda, \mu}^{-} .
$$

Now, the fact that $u_{n} \in N_{\lambda, \mu}^{+}$and the uniqueness for $s_{n}^{+}$imply that $s_{n}^{+}=1$. Thus, $\bar{s}_{n}>1$. 
Now, the fact that $\phi_{u_{n}}^{\prime \prime}(s)>0, s \in\left(0, \bar{s}_{n}\right)$ yields that $\phi_{u_{n}}^{\prime}(s)$ is strongly increasing. Combining with $\phi_{u_{n}}^{\prime}(1)=0$ and $1<\bar{s}_{n}$, we immediately obtain that $\phi_{u_{n}}^{\prime}(s) \leq 0, s \in[0,1]$, namely,

$$
s\left\|u_{n}\right\|_{X^{\alpha}}^{2}-\lambda s \int_{\mathbb{R}}\left|u_{n}\right|^{2} d t-s^{q-1} \int_{\mathbb{R}} b(t)\left|u_{n}\right|^{q} d t-\mu \int_{\mathbb{R}} h(t) \cdot u_{n}(t) d t \leq 0, \quad s \in[0,1] .
$$

Passing to the limit on the above inequality and taking into account (3.17)-(3.19), we get

$$
\begin{aligned}
& \phi_{\bar{u}}^{\prime}(s)=s\|\bar{u}\|_{X^{\alpha}}^{2}-\lambda s \int_{\mathbb{R}}|\bar{u}|^{2} d t-s^{q-1} \int_{\mathbb{R}} b(t)|\bar{u}|^{q} d t-\mu \int_{\mathbb{R}} h(t) \cdot \bar{u}(t) d t<0, \\
& \quad s \in(0,1] .
\end{aligned}
$$

On the other hand, by (3.24), $\phi_{\bar{u}}^{\prime}\left(s_{1}^{+}\right)=0$, which combined with (3.25) implies that $s_{1}^{+}>1$. Consequently, $\bar{s}>s_{1}^{+}>1$. Again, in terms of (3.24) and (3.20), we have

$$
C_{\lambda, \mu}^{+}=\inf _{u \in N_{\lambda, \mu}^{+}} I_{\lambda, \mu}(u) \leq I_{\lambda, \mu}\left(s_{1}^{+} \bar{u}\right)=\inf _{0 \leq s \leq \bar{s}} \phi_{\bar{u}}(s) \leq \phi_{\bar{u}}(1)=I_{\lambda, \mu}(\bar{u})<\lim _{n \rightarrow \infty} I_{\lambda, \mu}\left(u_{n}\right)=C_{\lambda, \mu}^{+},
$$

which is a contradiction. Thus, $\lim _{\bar{n} \rightarrow \infty}\left\|u_{n}\right\|_{X^{\alpha}}=\|\bar{u}\|_{X^{\alpha}}$, which yields that $u_{n} \rightarrow \bar{u}$ in $X^{\alpha}$ because $X^{\alpha}$ is a Hilbert space taking into account that $u_{n} \rightarrow u$ in $X^{\alpha}$.

Now, by (3.17)-(3.18), it is easy to see that

$$
I_{\lambda, \mu}(\bar{u})=\lim _{n \rightarrow \infty} I_{\lambda, \mu}\left(u_{n}\right)=C_{\lambda, \mu}^{+}
$$

On the other hand, from $I_{\lambda, \mu}^{\prime}\left(u_{n}\right) u_{n}=0$ and $\left(I_{\lambda, \mu}^{\prime}\left(u_{n}\right) u_{n}\right)^{\prime}\left(u_{n}\right)>0$ together with $u_{n} \rightarrow \bar{u}$ in $X^{\alpha}$, we immediately have

$$
I_{\lambda, \mu}^{\prime}(\bar{u}) \bar{u}=0, \quad\left(I_{\lambda, \mu}^{\prime}(\bar{u}) \bar{u}\right)^{\prime} \bar{u} \geq 0 .
$$

Again, by Lemma 3.2, $I_{\lambda, \mu}(\bar{u})=C_{\lambda, \mu}^{+}<0$, and therefore, $\bar{u} \neq 0$. By Lemma 2.6, $N_{\lambda, \mu}^{0}=\emptyset$. Hence, $\left(I_{\lambda, \mu}^{\prime}(\bar{u}) \bar{u}\right)^{\prime} \bar{u}>0$. This means that $\bar{u} \in N_{\lambda, \mu}^{+}$. Hence, $I_{\lambda, \mu}$ arrives at its minimum in $\bar{u} \in N_{\lambda, \mu}^{+}$on $N_{\lambda, \mu}^{+}$.

Lemma 3.5 Assume that hypotheses $\left(V_{0}\right)$ and $\left(H_{1}\right)$ hold. In addition,

1. if $h=0$, then $I_{\lambda, \mu}$ achieves its minimum at some $\bar{u} \in N_{\lambda, \mu}^{-}$on $N_{\lambda, \mu}^{-}$for $-\infty<\lambda<\lambda_{0}$.

2. if $h \neq 0$, then $I_{\lambda, \mu}$ achieves its minimum at some $\bar{u} \in N_{\lambda, \mu}^{-}$on $N_{\lambda, \mu}^{-}$for $-\infty<\lambda<\lambda_{0}$, $0<\mu<\mu_{\lambda} / 2$.

Proof First, by Lemma 3.3, $C_{\lambda, \mu}^{-}>0$. Now, we are going to make an argument similar to that in Lemma 3.4. Let $\left\{u_{n}\right\} \subset N_{\lambda, \mu}^{-}$be a minimizing sequence of $I_{\lambda, \mu}$ on $N_{\lambda, \mu}^{-}$, namely, $\lim _{n \rightarrow \infty} I_{\lambda, \mu}\left(u_{n}\right)=\inf _{u \in N_{\lambda, \mu}^{-}} I_{\lambda, \mu}(u):=C_{\lambda, \mu}^{-}$. Moreover, $\left\{u_{n}\right\}$ is bounded in $X^{\alpha}$, and therefore, there exists $\bar{u} \in X^{\alpha}$ such that $u_{n} \rightarrow \bar{u}$ in $X^{\alpha}$. The same reason as in Lemma 3.4 implies that (3.17)-(3.18) still hold.

Now, we show that $\lim _{n \rightarrow \infty}\left\|u_{n}\right\|_{X^{\alpha}}=\|\bar{u}\|_{X^{\alpha}}$. In fact, if not, then

$$
\|\bar{u}\|_{X^{\alpha}}<\lim _{n \rightarrow \infty}\left\|u_{n}\right\|_{X^{\alpha}}
$$


We claim that $\bar{u} \neq 0$. In fact, by $u_{n} \in N_{\lambda, \mu}$, it follows from (2.1) and (2.3) that

$$
I_{\lambda, \mu}\left(u_{n}\right)=-\frac{1}{2}\left(\left\|u_{n}\right\|_{X^{\alpha}}^{2}-\lambda \int_{\mathbb{R}}\left|u_{n}\right|^{2} d t\right)+\left(1-\frac{1}{q}\right) \int_{\mathbb{R}} b(t)\left|u_{n}\right|^{q} d t
$$

Hence,

$$
\begin{aligned}
\left(1-\frac{1}{q}\right) \int_{\mathbb{R}} b(t)\left|u_{n}\right|^{q} d t & =I_{\lambda, \mu}\left(u_{n}\right)+\frac{1}{2}\left(\left\|u_{n}\right\|-\lambda \int_{\mathbb{R}}\left|u_{n}\right|^{2} d t\right) \\
& \geq C_{\lambda, \mu}^{-}+\frac{1}{2}\left(1-\lambda S_{2}^{2}\right)\left\|u_{n}\right\|_{X^{\alpha}} .
\end{aligned}
$$

Passing to the limit on the above inequality as $n \rightarrow \infty$ and in view of (3.18), (3.26), we have

$$
\left(1-\frac{1}{q}\right) \int_{\mathbb{R}} b(t)|\bar{u}|^{q} d t>C_{\lambda, \mu}^{-}+\frac{1}{2}\left(1-\lambda S_{2}^{2}\right)\|\bar{u}\|_{X^{\alpha}}
$$

From $C_{\lambda, \mu}^{-}>0$, it follows that $\int_{\mathbb{R}} b(t)|\bar{u}|^{q} d t>0$, and therefore, $\bar{u} \neq 0$.

Now, by the proof of Lemma 3.4 and with an argument similar to (3.2) and (3.9), we know that there exists unique $s^{-}>0$ such that

$$
s^{-} \bar{u} \in N_{\lambda, \mu}^{-}, \quad I_{\lambda, \mu}\left(s^{-} \bar{u}\right)=\sup _{s \geq 0} I_{\lambda, \mu}(s \bar{u})
$$

Similarly, owing to $u_{n} \in N_{\lambda, \mu}^{-}$, we also have that $I_{\lambda, \mu}\left(u_{n}\right)=\sup _{s \geq 0} I_{\lambda, \mu}\left(s u_{n}\right)$, and therefore,

$$
I_{\lambda, \mu}\left(s^{-} u_{n}\right) \leq I_{\lambda, \mu}\left(u_{n}\right)
$$

Once again, (3.17)-(3.18) and (3.26) imply that

$$
I_{\lambda, \mu}\left(s^{-} \bar{u}\right)<\lim _{n \rightarrow \infty} I_{\lambda, \mu}\left(s^{-} u_{n}\right)
$$

Hence, by (3.27)-(3.29), we immediately have

$$
C_{\lambda, \mu}^{-}=\inf _{u \in N_{\lambda, \mu}^{-}} I_{\lambda, \mu}(u) \leq I_{\lambda, \mu}\left(s^{-} \bar{u}\right)<\lim _{n \rightarrow \infty} I_{\lambda, \mu}\left(s^{-} u_{n}\right) \leq \lim _{n \rightarrow \infty} I_{\lambda, \mu}\left(u_{n}\right)=C_{\lambda, \mu}^{-},
$$

a contradiction. Hence, $\lim _{n \rightarrow \infty}\left\|u_{n}\right\|_{X^{\alpha}}=\|\bar{u}\|_{X^{\alpha}}$. By an argument similar to that in the proof of Lemma 3.4, we obtain that $I_{\lambda, \mu}$ achieves its minimum at $\bar{u}$ on $N_{\lambda, \mu}^{-}$.

Now, we are in a position to show our first result on the existence of solution to (1.4).

Theorem 3.1 Assume that hypotheses $\left(V_{0}\right)$ and $\left(H_{1}\right)$ hold. If $h=0$, then problem (1.4) has at least one nontrivial weak solution for $-\infty<\lambda<\lambda_{0}$.

Proof Under the conditions assumed in this theorem, by Lemma 2.6, Lemma 3.1, Lemma 3.3, and Lemma 3.5, we know that $N_{\lambda, \mu}^{0}=\emptyset, N_{\lambda, \mu}^{-} \neq \emptyset$, and $I_{\lambda, \mu}$ achieves its minimum at some $\bar{u} \in N_{\lambda, \mu}^{-}$. Precisely, $I_{\lambda, \mu}(\bar{u})=\inf _{u \in N_{\lambda, \mu}^{-}} I_{\lambda, \mu}(u)=C_{\lambda, \mu}^{-}>0$. Because $N_{\lambda, \mu}^{-}$is an 
open subset in $N_{\lambda, \mu}$ and $\bar{u} \in N_{\lambda, \mu}^{-}$, there exists a ball $B(\bar{u}, r)$ centered at $\bar{u}$ with radius $r$ satisfying $B(\bar{u}, r) \cap N_{\lambda, \mu} \subset N_{\lambda, \mu}^{-}$. Thus

$$
I_{\lambda, \mu}(\bar{u})=\inf _{u \in N_{\lambda, \mu}^{-}} I_{\lambda, \mu}(u)=\inf _{u \in N_{\lambda, \mu}^{-} \cap B(\bar{u}, r)} I_{\lambda, \mu}(u)=\inf _{u \in N_{\lambda, \mu} \cap B(\bar{u}, r)} I_{\lambda, \mu}(u) .
$$

Hence, by Lemma 2.5 and taking account of the fact that $N_{\lambda, \mu}^{0}=\emptyset$, we immediately obtain that $\bar{u}$ is a nontrivial weak solution of (1.4). This completes the proof.

We give another result on the multiplicity of solutions to (1.4).

Theorem 3.2 Assume that hypotheses $\left(V_{0}\right)$ and $\left(H_{1}\right)$ hold. If $h \neq 0$, then problem (1.4) has at least two nontrivial weak solutions for $-\infty<\lambda<\lambda_{0}, 0<\mu<\mu_{\lambda} / 2$.

Proof Under these conditions, by Lemma 2.6 and Lemmas 3.1-3.5, we know that $N_{\lambda, \mu}^{0}=$ $\emptyset, N_{\lambda, \mu}^{-} \neq \emptyset, N_{\lambda, \mu}^{+} \neq \emptyset$. Moreover, there exist $\bar{u}_{1} \in N_{\lambda, \mu}^{-}, \bar{u}_{2} \in N_{\lambda, \mu}^{+}$such that

$$
I_{\lambda, \mu}\left(\bar{u}_{1}\right)=\inf _{u \in N_{\lambda, \mu}^{-}} I_{\lambda, \mu}(u)>0, \quad I_{\lambda, \mu}\left(\bar{u}_{2}\right)=\inf _{u \in N_{\lambda, \mu}^{+}} I_{\lambda, \mu}(u)<0 .
$$

Because $N_{\lambda, \mu}^{+}, N_{\lambda, \mu}^{-}$are two open subsets in $N_{\lambda, \mu}$ with $N_{\lambda, \mu}^{+} \cap N_{\lambda, \mu}^{-}=\emptyset$ and $N_{\lambda, \mu}^{0}=\emptyset$, making an argument similar to that in the proof of Theorem 3.1, we can deduce that $\bar{u}_{1}, \bar{u}_{2}$ are two nontrivial solutions of problem (1.4). The proof is complete.

\section{Conclusion}

In this paper, the authors investigate the existence and multiplicity of solutions for fractional Hamiltonian systems (1.4). Because the energy functional corresponding to system (1.4) is unbounded from below, it is invalid to try finding a critical point by applying the direct method of minimization to the energy functional. To overcome this difficulty, we introduce the Nehari manifold $N_{\lambda, \mu}$ and split it into three parts $N_{\lambda, \mu}^{-}, N_{\lambda, \mu}^{+}$, and $N_{\lambda, \mu}^{0}$. By looking for minimizer of the energy functional on $N_{\lambda, \mu}^{-}$and $N_{\lambda, \mu}^{+}$respectively and with the help of more analysis techniques, the authors establish some results on the existence and multiplicity of solutions to the above problem.

Acknowledgements

The authors wish to thank anonymous referees for their valuable suggestions.

Funding

This work was supported by the National Natural Science Foundation of China (No. 11601139).

Abbreviations

Not applicable.

Availability of data and materials

Not applicable.

Competing interests

The authors declare that they have no competing interests.

Authors' contributions

All authors contributed equally to this paper. All authors read and approved the final manuscript.

Publisher's Note

Springer Nature remains neutral with regard to jurisdictional claims in published maps and institutional affiliations.

Received: 6 January 2019 Accepted: 7 April 2019 Published online: 15 April 2019 


\section{References}

1. Hilfer, R.: Applications of Fractional Calculus in Physics. World Science, Singapore (2000)

2. Agrawal, O., Tenreiro Machado, J., Sabatier, J.: Fractional Derivatives and Their Application: Nonlinear Dynamics. Springer, Berlin (2004)

3. Kilbas, A., Srivastava, H., Trujillo, J.: Theory and Applications of Fractional Differential Equations. North-Holland Mathematics Studies, vol. 204. Elsevier, Amsterdam (2006)

4. Podlubny, I.: Fractional Differential Equations. Academic Press, New York (1999)

5. Diethelm, K.: The Analysis of Fractional Differential Equations. Springer, Heidelberg (2010)

6. Agarwal, R., Benchohra, N., Hamani, S.: A survey on existence result for boundary value problems of nonlinear fractional differential equations and inclusions. Acta Appl. Math. 109, 973-1033 (2010)

7. Agarwal, R., Hristova, S., O'Regan, D.: Iterative techniques for the initial value problem for Caputo fractional differential equations with non-instantaneous impulses. Appl. Math. Comput. 334, 407-421 (2018)

8. Ahmad, B., Luca, R.: Existence of solutions for sequential fractional integro-differential equations and inclusions with nonlocal boundary conditions. Appl. Math. Comput. 339, 516-534 (2018)

9. Wang, J., Fečkan, M., Zhou, Y.: A survey on impulsive fractional differential equations. Fract. Calc. Appl. Anal. 19(4), 806-831 (2016)

10. Jiao, F., Zhou, Y.: Existence results for fractional boundary value problems via critical point theory. Int. J. Bifurc. Chaos 22(4), 1-17 (2012)

11. Chai, G., Chen, J.: Existence of solutions for impulsive fractional boundary value problems via variational method. Bound. Value Probl. 2017, 23 (2017)

12. Nyamoradi, N., Rodríguez-López, R.: On boundary value problems for impulsive fractional differential equations. Appl. Math. Comput. 271, 874-892 (2015)

13. Chai, G., Liu, W.: Existence of solutions for the fractional Kirchhoff equations with sign-changing potential. Bound. Value Probl. 2018, 125 (2018)

14. Li, D., Chen, F., An, Y.: Existence and multiplicity of nontrivial solutions for nonlinear fractional differential systems with p-Laplacian via critical point theory. Math. Methods Appl. Sci. 41(8), 3197-3212 (2018)

15. Zhao, Y., Chen, H., Xu, C.: Nontrivial solutions for impulsive fractional differential equations via Morse theory. Appl. Math. Comput. 307, 170-179 (2017)

16. Averna, D., Tersian, S., Tornatore, E.: On the existence and multiplicity of solutions for Dirichlet's problem for fractional differential equations. Fract. Calc. Appl. Anal. 19(1), 253-266 (2016)

17. Nyamoradi, N.: Multiplicity of nontrivial solutions for boundary value problem for impulsive fractional differential inclusions via nonsmooth critical point theory. Fract. Calc. Appl. Anal. 18(6), 1470-1491 (2015)

18. Saoudi, K., Agarwal, P., Kumam, P., Ghanmi, A., Thounthong, P.: The Nehari manifold for a boundary value problem involving Riemann-Liouville fractional derivative. Adv. Differ. Equ. 2018, 263 (2018)

19. Ge, B., Lu, J., Zhao, T., Zhou, K.: Superlinear fractional boundary value problems without the Ambrosetti-Rabinowitz condition. Electron. J. Differ. Equ. 2018, 85 (2018)

20. Nyamoradi, N., Zhou, Y., Ahmad, B., Alsaedi, A.: Variational methods for Kirchhoff type problems with tempered fractional derivative. Electron. J. Differ. Equ. 2018, 34 (2018)

21. Khaliq, A., ur Rehman, M.: On variational methods to non-instantaneous impulsive fractional differential equation. Appl. Math. Lett. 83, 95-102 (2018)

22. Heidarkhani, S., Cabada, A., Afrouzi, G.A., Moradi, S., Caristi, G.: A variational approach to perturbed impulsive fractional differential equations. J. Comput. Appl. Math. 341, 42-60 (2018)

23. Wang, Y., Liu, Y., Cui, Y.: Infinitely many solutions for impulsive fractional boundary value problem with p-Laplacian. Bound. Value Probl. 2018, 94 (2018)

24. Torres, C.: Existence of solutions for fractional Hamiltonian systems. Electron. J. Differ. Equ. 2013, 259 (2013)

25. Amado, M., Torres, C.: Multiplicity of solutions for fractional Hamiltonian systems with Liouville-Weyl fractional derivatives. Fract. Calc. Appl. Anal. 18(4), 875-890 (2015)

26. Zhang, Z., Yuan, R.: Variational approach to solutions for a class of fractional Hamiltonian systems. Math. Methods Appl. Sci. 37, 1873-1883 (2014)

27. Zhou, Y., Zhang, L.: Existence and multiplicity results of homoclinic solutions for fractional Hamiltonian systems. Comput. Math. Appl. 73, 1325-1345 (2017)

28. Xu, J., O’Regan, D., Zhang, K.: Multiple solutions for a class of fractional Hamiltonian systems. Fract. Calc. Appl. Anal. 18(1), 48-53 (2015)

29. Zhang, Z., Yuan, R.: Existence of solutions to fractional Hamiltonian systems with combined nonlinearities. Electron. J. Differ. Equ. 2016, 40 (2016)

30. Hu, Z., Liu, W., Chen, T.: The existence of a ground state solution for a class of fractional differential equation with p-Laplacian operator. Bound. Value Probl. 2016, 45 (2016)

31. Torres, C.: Tempered fractional differential equation: variational approach. Math. Methods Appl. Sci. 2017(40), 4962-4973 (2017)

32. Poincaré, H.: Les Méthodes Nouvelles de la Mécanique Céleste. Gauthier-Villars, Paris (1892, 1893, 1899)

33. Omana, W., Willem, M.: Homoclinic orbits for a class of Hamiltonian systems. Differ. Integral Equ. 5(5), 1115-1120 (1992)

34. Rabinowitz, P.H.: Homoclinic orbits for a class of Hamiltonian systems. Proc. R. Soc. Edinb., Sect. A 114, 33-38 (1990)

35. Torres, C.: Existence and concentration of solution for a class of fractional Hamiltonian systems with subquadratic potential. Proc. Indian Acad. Sci. Math. Sci. 128, Article ID 50 (2018)

36. Torres, C., Zhang, Z.: Concentration of ground state solutions for fractional Hamiltonian systems. Topol. Methods Nonlinear Anal. 50(2), 623-642 (2017)

37. Mawhin, J., Willem, M.: Critical Point Theory and Hamiltonian Systems. Springer, New York (1989)

38. Ervin, V., Roop, J.: Variational formulation for the stationary fractional advection dispersion equation. Numer. Methods Partial Differ. Equ. 22, 58-76 (2006)

39. Chang, K.C.: Methods in Nonlinear Analysis. Springer Monographs in Mathematics. Springer, Berlin (2005) 